\title{
The relationship of tooth shade and skin tone and its influence on the smile attractiveness
}

\author{
Bianca Di Murro DDS ${ }^{1}$ ( ) | Gianni Gallusi DDS, PhD ${ }^{1}$ | Roberto Nardi DDS ${ }^{1}$ | \\ Antonio Libonati DDS, PhD ${ }^{2}$ | Vincenzo Angotti DDS ${ }^{1}$ | Vincenzo Campanella MD, DDS ${ }^{1}$
}

${ }^{1}$ Department of Clinical and Translational Medicine, Tor Vergata University of Rome, Rome, Italy

${ }^{2}$ Department of Surgical Sciences, Catholic University of Our Lady of Good Counsel of Tirane, Tirana, Albania

\section{Correspondence}

Bianca Di Murro, Department of Clinical and Translational Medicine, Tor Vergata University of Rome, 1 Montpellier Street, 00133, Rome, Italy.

Email: biancadimurro@hotmail.it

\begin{abstract}
Objective: The purpose of this clinical study was to determine if skin tone and tooth color had the same influence on smile attractiveness.

Materials and Methods: A woman's smile photograph was digitally manipulated to create a range of images with varying colors. The skin shade was modified to create four tones (p1: light, p2: light medium, p3: medium dark, p4: dark) using the L'Oreal True Illusion shade as a guide. The tooth shade was modified in four different tones: A1, A2, A3, A4 using the VITAPAN Classical shade guide to produce 16 images. A sample of 328 participants rated each image for attractiveness by means of a visual analog scale (VAS). Comparison among groups was performed with a 2-way ANOVA adjusted for multiple comparisons with the Bonferroni test $(\alpha=.05)$.

Results: Image (p3-a1) showed the highest VAS values, while image (p1-a4) obtained the lowest, (Bonferroni Test: a1 vs a2, a3, a4 $P<.001$; p3 vs p1, p2, p4 $P<.001$ ). Analysis performed for age, sex, level of education, and laypeople/dentists were not statistically significant $(P>.05)$.

Conclusions: Variations in tooth and skin tone can significantly influence the perception of smile attractiveness. In the tested conditions, a brighter tooth shade significantly affected the attractiveness of the smile independently from skin tone.

Clinical Significance: Understanding patient and dentist perception of the attractiveness of a smile with the important role-played by skin tone, may help clinicians to better identify teeth shade, helping delivery of tailored prostheses and esthetic restorations.
\end{abstract}

\section{KEYWORDS}

chroma, esthetics, skin tone, smile attractiveness, tooth color, value

\section{1 | INTRODUCTION}

Eyes, smile, and the mouth in general are the areas most associated with the attractiveness of the face. ${ }^{1-3}$ Smile is an important means of communications and facial expression. Many patients go to the dentist to improve and correct their smile, in order to achieve a more pleasant and natural appearance. The dentist can meet and satisfy the patient's esthetic needs by modifying the morphological and chromatic characteristics (shape and color) of the teeth, adopting increasingly effective techniques of adhesive ${ }^{4,5}$ and restorative dentistry. ${ }^{6-8}$

Dissatisfaction about the smile and tooth color has been reported to be a major concern among adults. ${ }^{9,10}$ Tooth color was considered a major factor in relation to dental esthetics and shape and color were judged as key parameters to evaluate the attractiveness of a smile. ${ }^{11}$ 
Various criteria have been evaluated for tooth shape including proportion and shape of the maxillary central incisors ${ }^{12-14}$ and symmetry of the midline. ${ }^{15}$ However, universal guidelines are lacking for tooth color.

Studies focusing on the relationship between skin tone and tooth color are sparse and they have not considered overall esthetic attractiveness. Haralur et $\mathrm{al}^{16}$ and Vadavadagi et $\mathrm{al}^{17}$ reported that tooth color was correlated with skin shade, recommending evaluating skin shade as a guide in the choice of tooth color. This recommendation has been supported by other studies ${ }^{18,19}$ reporting that people with a darker skin color have teeth with higher value and people with lighter skin color have darker teeth. In contrast, according to another study, ${ }^{20}$ no relationship has been established between skin and tooth shade. Only few articles ${ }^{21,22}$ evaluated the perception of smile attractiveness, in relation to the color. Sabherwal et al, ${ }^{21}$ asked dentists and laypeople to evaluate 24 images created by matching four skin tones with six tooth shades. Labban et $\mathrm{al}^{22}$ manipulated two images representing a male and a female smile with four different skin tones and six different tooth shades, for a total of 48 modified images, participants were enrolled in shopping malls.

Skin tone is difficult to define because of the lack of a wellcategorized reference scale or guide, while tooth color is better defined. The Fitzpatrick guide ${ }^{23}$ is more related to dermatology and orientated in defining a phototype, categorizing skin damage after sun exposure, without valid reference samples. Different studies, ${ }^{18,19,24}$ categorized skin tone, according to different cosmetic indexes, used to compare skin color with samples, such as NIVEA, LAKME, or L'Oreal.

The purpose of this clinical study was to evaluate how tooth and skin tone can influence the perception of the smile attractiveness. The research hypothesis was that skin tone and tooth color had the same influence on smile attractiveness.

\section{2 | MATERIALS AND METHODS}

The authors designed and implemented a cross sectional survey, conducted at the Department of Restorative Dentistry, University of Rome "Tor Vergata" and approved by the local Institution Review Board (ref: 175/18). A consent was obtained from each participant prior to being enrolled in this survey. The study sample was composed of a population of general dentists and Italian laypeople derived from patients presenting at the Tor Vergata General Hospital in 2018. Exclusion criteria were color blindness (tested by the Ishihara test) (Figure 1) and non-Italian nationality.

The authors selected a young woman ( 26 years) with a smile exhibiting good dental alignment and tooth size symmetry. A standardized frontal view smile photograph showing teeth, lips, and surrounding skin in ambient light was made with a digital camera (D7000; Nikon Corporation). The nose and chin were cropped out to reduce the number of confounding variables. The photographic image was digitally modified (Adobe Photoshop CS5; Adobe) to create a
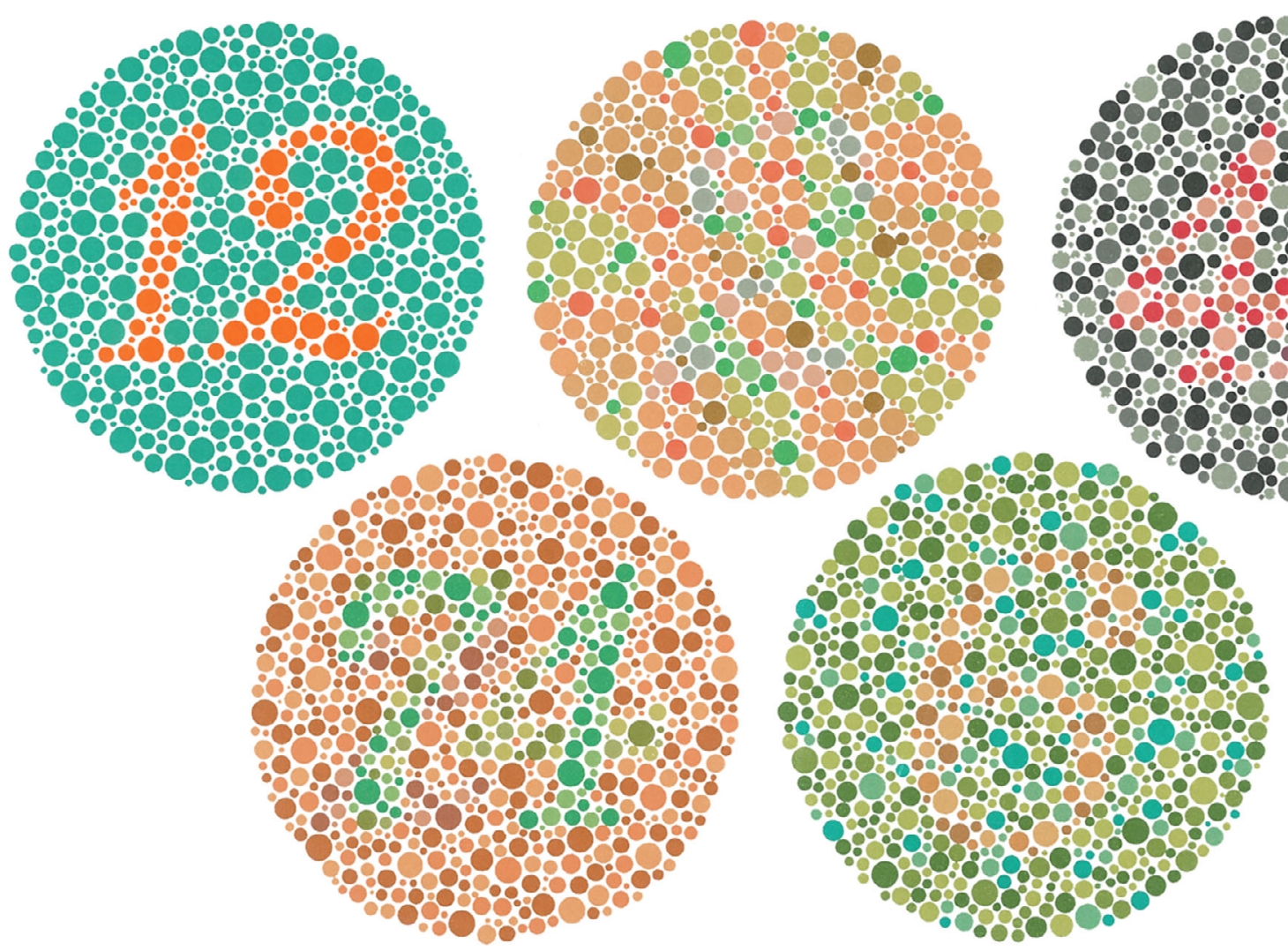

FIGURE 1 Ishihara test 
range of images with varying skin and tooth shades (Figure 2). The selected smile image was edited into Photoshop in order to separate teeth, gums, lips, and skin into four different levels. In this manner, it was then possible to alter hue, vividness, saturation, and light to match the reference shade scales and produce the complete set of photographs with all variants.

The skin shade was altered to create four tones (p1: light, p2: light medium, p3: medium dark, p4: dark) selected from the L'Oreal True Illusion compact makeup shades (L'Oreal). ${ }^{19}$ This guide has 15 shades from which 4 were selected to represent the main range of complexions.

The tooth shade was modified to create four different tones (A1, $A 2, A 3$, and $A 4)$ using the hue $A$ selected from the Vita classical A1-D4 system (VITA Zahnfabrik, Bad Säckingen, Germany). ${ }^{25}$ This guide has four grouping of shapes (A1-A4: reddish-brownish; B1-B4: reddish-yellowish; C1-C4: greyish shades; D2-D4: reddish-grey).

Sixteen images were generated and each image was printed, named with an arbitrary two letters code, and randomly ordered in a sequence (blind selection from closed envelopes). The resulting randomized sequence of 16 images was the following order: ZC (p1-a3); JL (p1-a1); BZ (p2-a4); PO (p4-a2); XC (p2-a1); RV (p1-a2); JH (p3-a3); AX (p3-a1); NI (p2-a3); SW (p3-a2); NM (p4-a4); LS (p2-a2); AQ (p4-a1); IU (p1-a4); SE (p4-a3); AM (p3-a4). Image number $7(\mathrm{JH})$ was duplicated and renamed GB (p3-a3); this image was inserted at the end of the test image sequence to assess intra- examiner reliability; hence, participants viewed a total of 17 images.

At the beginning of the interview, the examiner recorded, on a separate sheet of paper, age (15-45, 45,1-65, 65,1-90 years), sex $(\mathrm{M} / \mathrm{F})$, level of education (middle school, high school, university), and skin tone of each participant ( $n=328 ; 179$ females and 149 males). Skin tone was evaluated from the forearm and categorized in four tones (light, light medium, medium dark, and dark) according to same scale used for the images.

A slide show presentation was prepared with images placed on a black background, arranged in the previously randomized order. Defective color vision was identified by the Ishihara test (Figure 1), placed on the first slide. The images were viewed on a $24.6 \mathrm{~cm}$ laptop screen (iPad; Apple Inc), set to a brightness of $50 \%$ and contrast of $100 \%$, while the participants rated the images. A blue screen appeared for 5 seconds between each image. The slides appeared in the same sequence for all participants. Participants rated the images without conferring with others. Participants rated each image for attractiveness by using a visual analog scale (VAS), that consisted in a $100 \mathrm{~mm}$ line from point $0=$ extremely unattractive to point $10=$ extremely attractive. Each VAS was printed on a different sheet of paper for a total of 17 pages. Every participant was asked to mark an " $X$ " on the line answering to the question "How attractive do you consider this smile?". Every interview was made in a well-lighted location with natural and artificial light between 9 AM and 4 PM

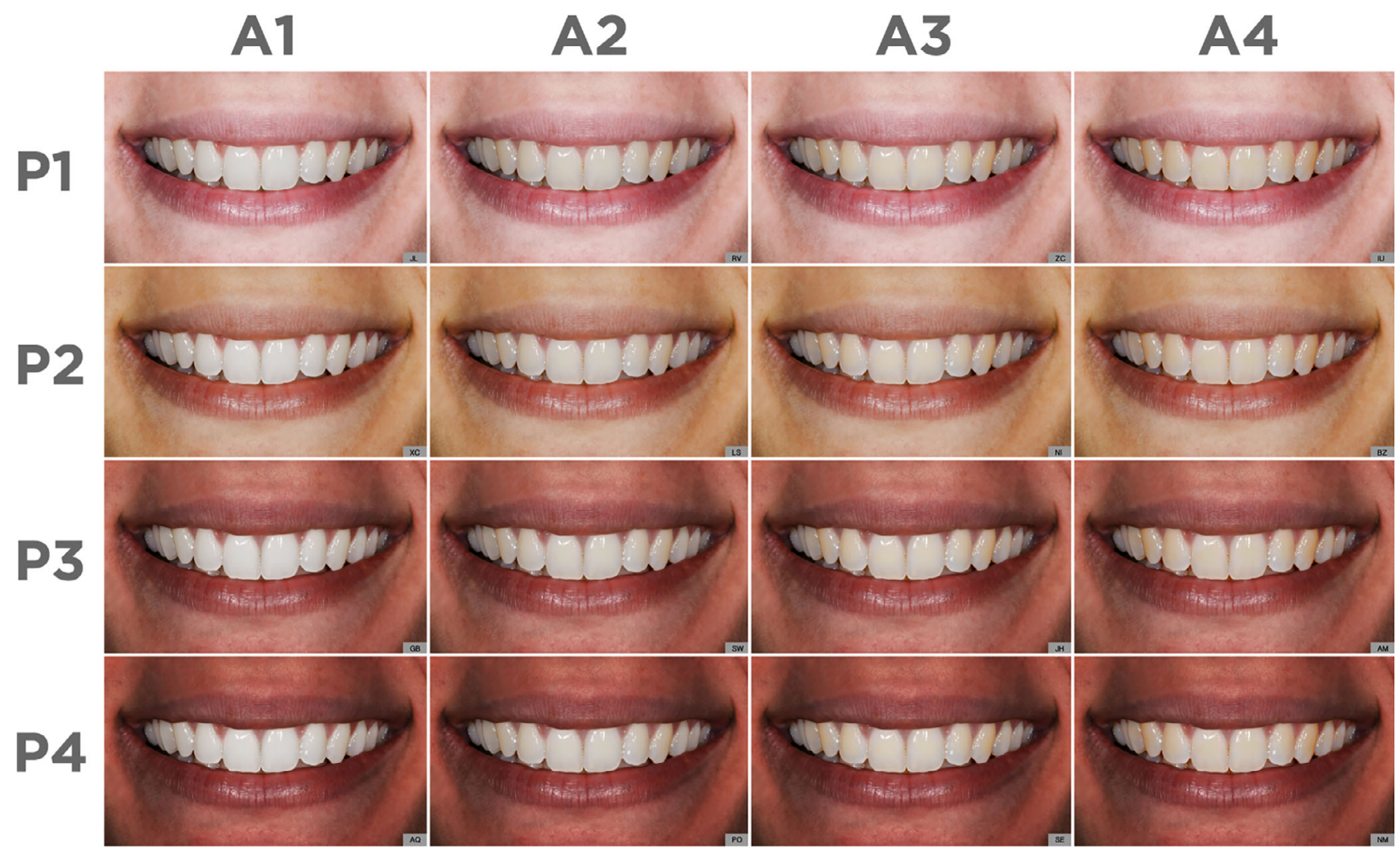

FIGURE 2 Tested images, obtained from the combination of different teeth shade (a1, a2, a3, and a4 of VITAPAN Classical a1-d4) and skin tone (p1, p2, p3, p4 of L'Oreal True Illusion compact makeup shades) 
TABLE 1 Descriptives of sample $(\mathrm{N}=328)$ by sex

\begin{tabular}{|c|c|c|c|c|c|c|c|c|c|c|}
\hline \multicolumn{3}{|c|}{ Characteristic } & \multicolumn{3}{|c|}{ Education level } & Dentist (\%) & \multicolumn{4}{|c|}{ Skin tone } \\
\hline $\mathrm{F}$ & 179 & $45 \pm 18$ & $21(12)$ & $60(33)$ & $98(54)$ & $31(17)$ & $48(27)$ & $89(52)$ & $37(21)$ & $5(1)$ \\
\hline$M$ & 149 & $44 \pm 18$ & $20(13)$ & $44(29)$ & $85(57)$ & $40(27)$ & $29(19)$ & $72(50)$ & $44(29)$ & $4(1)$ \\
\hline
\end{tabular}

\begin{tabular}{|llllll|}
\hline Image & Mean & $\boldsymbol{\sigma}$ & Skin Tone $(\mathbf{p})$ & Tooth Shade $(\mathrm{a})$ & Code $(10 \mathrm{p}+\mathrm{a})$ \\
\hline $\mathrm{AX}$ & 72 & 23 & $\mathrm{p} 3$ & $\mathrm{a} 1$ & 31 \\
\hline JL & 69 & 21 & $\mathrm{p} 1$ & $\mathrm{a} 1$ & 11 \\
\hline $\mathrm{AQ}$ & 69 & 23 & $\mathrm{p} 4$ & $\mathrm{a} 1$ & 41 \\
\hline XC & 69 & 23 & $\mathrm{p} 2$ & $\mathrm{a} 1$ & 21 \\
\hline SW & 65 & 23 & $\mathrm{p} 3$ & $\mathrm{a} 2$ & 32 \\
\hline LS & 63 & 24 & $\mathrm{p} 2$ & $\mathrm{a} 2$ & 22 \\
\hline PO & 58 & 26 & $\mathrm{p} 4$ & $\mathrm{a} 2$ & 42 \\
\hline RV & 53 & 25 & $\mathrm{p} 1$ & $\mathrm{a} 2$ & 12 \\
\hline SE & 52 & 25 & $\mathrm{p} 4$ & $\mathrm{a} 3$ & 43 \\
\hline JH & 50 & 23 & $\mathrm{p} 3$ & $\mathrm{a} 3$ & 33 \\
\hline NI & 50 & 24 & $\mathrm{p} 2$ & $\mathrm{a} 3$ & 23 \\
\hline NM & 44 & 26 & $\mathrm{p} 4$ & $\mathrm{a} 4$ & 44 \\
\hline AM & 43 & 24 & $\mathrm{p} 3$ & $\mathrm{a} 4$ & 34 \\
\hline BZ & 42 & 23 & $\mathrm{p} 2$ & $\mathrm{a} 4$ & 24 \\
\hline ZC & 38 & 24 & $\mathrm{p} 1$ & $\mathrm{a} 3$ & 13 \\
\hline IU & 25 & 26 & $\mathrm{p} 1$ & $\mathrm{a} 4$ & 14 \\
\hline
\end{tabular}

TABLE 2 Mean VAS (visual analogic scale) values, images were coded for identification by using a unique two letter combination (ie, AX), $\sigma=\mathrm{SD}$

Note: Skin Tone: P1: light, P2: light medium, P3: medium dark, P4: dark. Tooth Shade from the brightest to the darkest: A1, A2, A3 and A4 using the hue A (red-brown) of VITA classical A1-D4 shade guide. Last column reports image coding for Bonferroni test.

A total number of 328 participants were enrolled in this study: 179 females (58.7\%) and 149 males (41.3\%), with a mean age of 52.5 \pm 23.3 years (age range $=16-89$ years). Sample included 257 laypeople (78.3\%) and 71 general dentists (21.7\%), as shown in Table 1. Each participant was recorded for sex (M/F), skin tone (p1-4), age, level of education, and dentist ( $\mathrm{Y} / \mathrm{N})$.

\section{3 | STATISTICAL ANALYSIS}

A power calculation was based on the mean VAS values obtained from a previous pilot study, where 10 participants evaluated images resulted from the matching of one skin tone (p3) and 2 adjacent tooth shades $(\mathrm{a} 3, \mathrm{a} 4)(\mathrm{p} 3-\mathrm{a} 3=43 \pm 23 \mathrm{~mm}, \mathrm{p} 3-\mathrm{a} 4=49 \pm 23 \mathrm{~mm}$, respectively).

Having HO: VAS $=43$ and $\mathrm{H} 1$ : VAS $=49$ with a constant SD of 23 and a $16 \%$ mean difference, the sample was calculated to be at least 230 participants for a $5 \%$ alpha error and $94 \%$ power. Intraexaminer reliability was high with a Cohen's Kappa of 0.86. Examiner reliability was evaluated with six multiple choices questions on the conduction of the test.
All data were initially entered into a database (Excel; Microsoft Corp) and analyzed with statistical software (SPSS v20.0; SPSS Inc). Descriptive statistics consisted of the mean and SDs for parameters with normal distributions (after confirmation with histograms and the Kolgomorov-Smirnov test), median and range (min.; max.) for variables with non-normal distributions.

VAS values of the 16 tested images were compared with a multiple comparison ANOVA test plus Bonferroni post-hoc test for sex $(\mathrm{M} / \mathrm{F})$, level of education (Middle, High, University), dentist $(\mathrm{Y} / \mathrm{N})$ and Age range (1:15-45; 2:45.1-65; 3:65.1-90). In addition, a chi-square test was used to evaluate most chosen images. The Pearson correlation coefficient was used to evaluate intraclass correlation ( $\alpha=.05)$.

\section{4 | RESULTS}

Image $A X(p 3-a 1=31)$ showed the highest VAS values $(72 \pm 23 \mathrm{~mm})$, while image IU ( 1 1-a4 = 14) obtained the lowest $(25 \pm 26 \mathrm{~mm}$ ) with all values shown in Table 2. Bonferroni $\mathrm{p}$ results are shown in Table 3 and in Figure 3 it is shown that independently from skin tone, the preferred tooth shade is always a1 (the brighter shade) over all others. 
TAB LE 3 Mean VAS (visual analogic scale) and SD for images coded (10p $+a$ ) with p:1-4 and a:1-4 and the $P$-values of the multiple tests of the Bonferroni test

\begin{tabular}{|c|c|c|c|c|c|c|c|c|c|c|c|c|c|c|c|c|}
\hline \multicolumn{2}{|l|}{ Descriptives } & \multicolumn{15}{|c|}{$P$-value of multiple comparison by Bonferroni Test } \\
\hline $70 \pm 21$ & 11 & - & & & & & & & & & & & & & & \\
\hline $53 \pm 25$ & 12 & .001 & & & & & & & & & & & & & & \\
\hline $38 \pm 24$ & 13 & .001 & .001 & & & & & & & & & & & & & \\
\hline $25 \pm 26$ & 14 & .001 & .001 & .001 & & & & & & & & & & & & \\
\hline $63 \pm 24$ & 22 & .081 & .001 & .001 & .001 & .262 & & & & & & & & & & \\
\hline $50 \pm 24$ & 23 & .001 & 1 & .001 & .001 & .001 & .001 & & & & & & & & & \\
\hline $42 \pm 23$ & 24 & .001 & .001 & 1 & .001 & .001 & .001 & .01 & & & & & & & & \\
\hline $72 \pm 23$ & 31 & 1 & .000 & .001 & .001 & 1 & .001 & .001 & .001 & & & & & & & \\
\hline $69 \pm 23$ & 41 & 1 & .000 & .001 & .001 & 1 & .184 & .001 & .001 & 1 & 1 & .000 & .001 & & & \\
\hline $58 \pm 26$ & 42 & .001 & 1 & .001 & .001 & .001 & 1 & .002 & .001 & .001 & .012 & .002 & .001 & .001 & & \\
\hline $53 \pm 25$ & 43 & .001 & 1 & .001 & .001 & .001 & .001 & 1 & .001 & .001 & .001 & 1 & .001 & .001 & .684 & \\
\hline $44 \pm 26$ & 44 & .001 & .000 & .131 & .001 & .001 & .001 & .233 & 1 & .001 & .001 & .225 & 1 & .001 & .001 & .001 \\
\hline
\end{tabular}

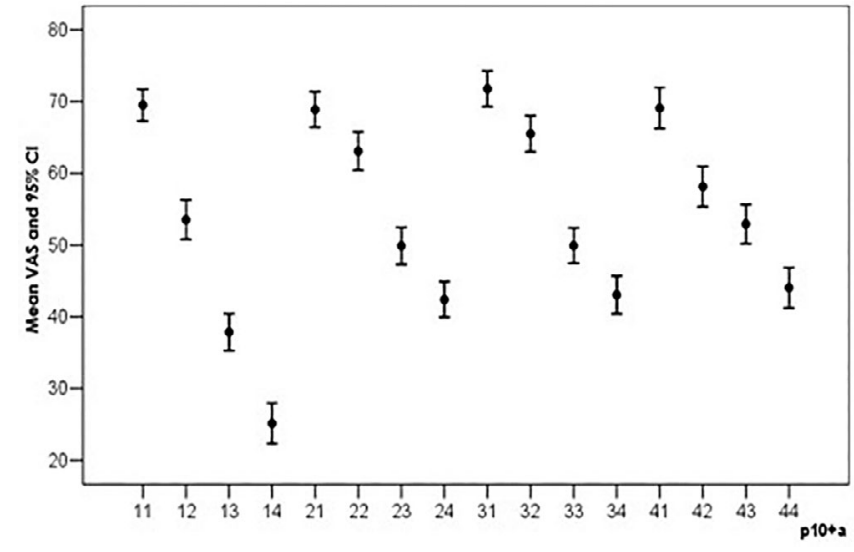

FIGURE 3 Error bar of mean VAS and $95 \% \mathrm{Cl}$ for skin tone ( $\mathrm{p}$ : 1-4) and teeth shade (a:1-4) and coded: 10 skin tone + tooth shade; $10 p+a$

On the contrary, lower VAS values for skin tone are always associated with tooth shade a4 (the darker shade).

Variable "Sex" did not affect significantly VAS values $(P=.755)$ as well as level of education $(P=.088)$, and Dentist $(P=.106)$. "Age range" gave VAS values significantly higher $(P=.010)$ accordingly with higher age. Nevertheless, these higher VAS values were not in contrast with the general trend and the most appreciated image was always AX (p3-a1 = 31) (Figure 4) for all age range (Chi-square, $P=.078$ ) as shown in Table 4. The Pearson correlation coefficient for VAS vs skin tone was $r=0.123, P<.01$, and VAS vs Tooth Shade was $r=-0.431$ with $P<0.01$.

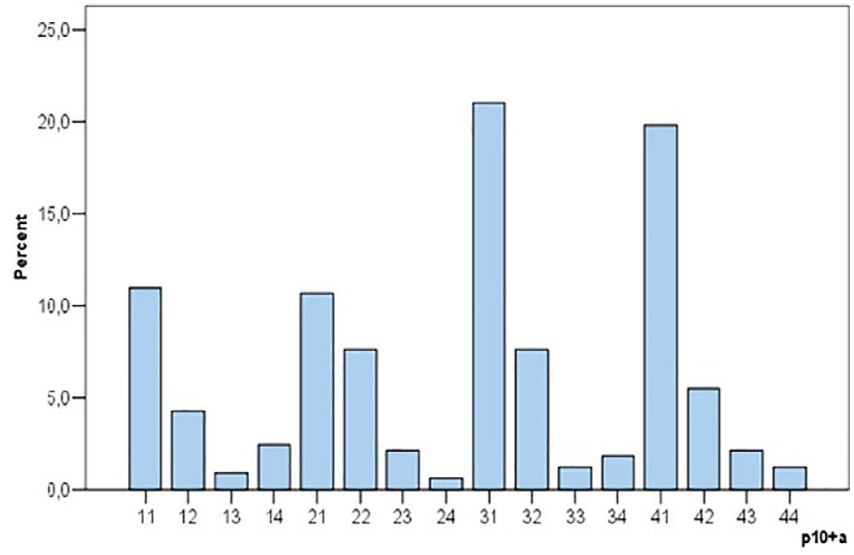

FIGURE 4 Bar chart of percentages of smile images chosen coded: 10 skin tone + tooth shade $(10 p+a)$

\section{5 | DISCUSSION}

The hypothesis of this study was that skin tone and tooth shade had the same influence on smile attractiveness. The results rejected this hypothesis: variations in tooth shade were more important than differences in skin tone. Results showed that independently from skin tone, the preferred tooth shade was always the brighter (higher VAS) and values for darker teeth were significantly lower.

In literature, there are no previous reviews or original studies to which our findings can be compared due to images used and composition of sample (laypeople and general dentists). 


\begin{tabular}{|lcccccc} 
& \multicolumn{2}{l}{ Range Age } & & & \\
\cline { 2 - 4 } coded 10p + a & 1: (15-45) & $2:(45.1-65)$ & $3:(65.1-90)$ & $\mathbf{n}^{\circ}$ choices & Percentage \% \\
\hline 11 & 21 & 9 & 6 & 36 & 11 \\
\hline 12 & 4 & 7 & 3 & 14 & 4.3 \\
\hline 13 & 3 & 0 & 0 & 3 & 0.9 \\
\hline 14 & 0 & 4 & 4 & 8 & 2.4 \\
\hline 21 & 19 & 9 & 7 & 35 & 10.7 \\
\hline 22 & 11 & 9 & 5 & 25 & 7.6 \\
\hline 23 & 6 & 1 & 0 & 7 & 2.1 \\
\hline 24 & 0 & 2 & 0 & 2 & 0.6 \\
\hline 31 & 39 & 18 & 12 & 69 & 21 \\
\hline 32 & 15 & 7 & 3 & 25 & 7.6 \\
\hline 33 & 1 & 1 & 2 & 4 & 1.2 \\
\hline 34 & 4 & 1 & 1 & 6 & 1.8 \\
\hline 41 & 35 & 21 & 9 & 65 & 19.8 \\
\hline 42 & 8 & 7 & 3 & 18 & 5.5 \\
\hline 43 & 2 & 5 & 0 & 7 & 2.1 \\
\hline 44 & 2 & 0 & 2 & 4 & 1.2 \\
\hline & 170 & 101 & 57 & 328 & \\
\hline
\end{tabular}

TABLE 4 Crosstabulation of 16 images coded $(10 p+a)$ vs range Age with $\mathrm{n}$ and percentage of the chosen images
In a similar study, conducted on a sample of 140 participants, Sabherwal et $\mathrm{al}^{21}$ reported that perception of smile attractiveness was different between "young" and "old" people. Our results are in contrast with Sabherwal et $a^{21}$ since no statistically significant differences based on participants age were found. The highest VAS values were found for images with darker skin and lighter tooth shade, this is also in contrast with Sabherwal et $\mathrm{al}^{21}{ }^{21}$ where this combination was seemed to be less attractive. This result could be related to the quality of the tested images. In the present study the images were prepared to appear as realistic as possible with great attention to replicate true skin tones and tooth shades (VITA and L'Oreal), while Sabherwal et $\mathrm{al}^{21}$ did not use any color reference. The VITA guide was selected because it is popular in Italy. ${ }^{26,27}$ The A shade was chosen as it has been reported from many authors the most frequently used and therefore the closer to natural teeth color shade. ${ }^{28}$

Labban et $\mathrm{al}^{22}$ evaluated 48 images of smiles and found out that gender had an influence on the perception of tooth shades: women participants preferred lighter shades if compared to men participants. Furthermore, lighter tooth shades were preferred for lighter skin tone and comparatively darker tooth shades were preferred for darker skin tone. Our results were in contrast with Labban et al ${ }^{22}$ since lighter tooth shades were always preferred independently of the skin tone and sex of the participant. In the present study, darker tooth shade (a3, a4) resulted more appreciated when combined with darker skin tone (p3, p4): due to the contrast with facial tissues, teeth appeared more brilliant (Figure 3). On the contrary, the combination of darker teeth (a4) and lighter skin tone ( $\mathrm{p} 1$ ), showed an exaggerated contrast and obtained the lowest values, this result is in accordance with Sabherwal et al. ${ }^{21}$

The matching of the results obtained in the survey with the skin shade of participants revealed that, despite only $23 \%$ of participants had p3 skin tone, image $A X(p 3-a 1=31)$ was the most appreciated. $A$ possible explanation to these findings is that participants have an idea of beauty different from their own physical aspect and skin characteristics. A hypothesis also supported by the fact that images which obtained the low VAS values resulted respectively p1-a3, p1-a4, and p2-a4, despite over $70 \%$ of participants presented light skin colors p2 (50.9\%) and $\mathrm{p} 1$ (23.7\%).

Main limitations of the study are represented by the nature of the enrolled sample (composed only by Italian people), and the absence of a well-categorized reference scale for skin tone.

Only Italian subjects were enrolled in this survey, since the population attending the Tor Vergata General Hospital is composed almost entirely by people with Italian nationality.

A lighter tooth shade enhances the smile attractiveness, with a1 combinations showing the highest VAS values independently from skin tone, according to the intra-class correlation analysis.

In patients with light colored skin, the choice of bright teeth appears important. Even if, in current literature, no significant association was detected between ethnicity and attitude about dental esthetics, ${ }^{29}$ future research should be orientated in investigating if these results may change in different cultures and with a more heterogeneous sample.

\section{6 | CONCLUSIONS}

In the tested conditions and within the limitations of the study a brighter tooth shade significantly affected ( $P$-value) the attractiveness of the smile independently from skin tone.

Variations in tooth and skin tone and their combination can significantly influence the perception of smile attractiveness. 


\section{ACKNOWLEDGMENTS}

We thank Professor Roberto Sorge (Department of Systems Medicine, University of Rome Tor Vergata, Rome, Italy) for all his help and support with the statistical analysis.

\section{CONFLICT OF INTEREST}

The authors report no conflict of interest. The authors do not have any financial interest in the companies whose materials are included in this article.

\section{ORCID}

\section{Bianca Di Murro (D) https://orcid.org/0000-0002-5342-5446}

\section{REFERENCES}

1. Rodrigues Cde D, Magnani R, Machado MS, et al. The perception of smile attractiveness. Angle Orthod. 2009;79:634-639.

2. Quinzi V, Scibetta ET, Marchetti E, et al. Analyze my face. J Biol Regul Homeost Agents. 2018;32:149-158.

3. Mummolo S, Nota A, Marchetti E, Padricelli G, Marzo G. The 3D tele motion tracking for the orthodontic facial analysis. Biomed Res Int. 2016;2016:1-6.

4. Ballesio I, Angotti V, Gallusi G, et al. Durability of adhesion between an adhesive and post-space dentin: push-out evaluation at one and six months. Int J Adhes Adhes. 2012;38:75-78.

5. Campanella V, Di Taranto V, Libonati A, et al. Indirect adhesive rehabilitation by cementation under pressure of a case of Dentinogenesis Imperfecta type II: follow-up after 13 years. Eur J Paediatr Dent. 2018 19:303-306.

6. Libonati A, Marzo G, Klinger FG, et al. Embryotoxicity assays for leached components from dental restorative materials. Reprod Biol Endocrinol. 2011;9:136.

7. Vadini M, De Angelis F, D'Amario M, et al. Conservative restorations of endodontically compromised anterior teeth in paediatric patients: physical and mechanical considerations. Eur J Paediatr Dent. 2012;13 263-267.

8. D'Amario $M$, De Angelis F, Vadini M, et al. Influence of repeated preheating procedure on mechanical properties of three resin composite. Oper Dent. 2015;40(2):181-189.

9. Van der Geld P, Oosterveld P, Van Heck G, et al. Smile attractiveness. Self-perception and influence on personality. Angle Orthod. 2007;77: 759-765.

10. Tin-Oo MM, Saddki N, Hassan N. Factors influencing patient satisfaction with dental appearance and treatments they desire to improve esthetics. BMC Oral Health. 2011;11:6.

11. Samorodnitzky-Naveh GR, Geiger SB, Levin L. Patients' satisfaction with dental esthetics. J Am Dent Assoc. 2007;138:805-808.

12. Machado AW, Moon W, Campos E, et al. Influence of spacing in the upper lateral incisor area on the perception of smile esthetics among orthodontists and laypersons. J World Fed Orthod. 2013:2:169-174.

13. Cooper GE, Tredwin CJ, Cooper NT, Petrie A, Gill DS. The influence of maxillary central incisor height-to-width ratio on perceived smile esthetics. Br Dent J. 2012:212:589-599.
14. Tay L, Herrera D, Bernabè E, et al. Esthetic perception towards different combinations of facial contours and upper incisor shape. Braz J Oral Sci. 2009;8:193-196.

15. Sharma N, Rosenstiel FS, Fields HW, et al. Smile characterization by U.S. white U.S. Asian Indian, and indian populations. J Prosthet Dent. 2012;107:327-335.

16. Haralur SB, Dibas AM, Almelhi NA, et al. The tooth and skin colour interrelationship across the different ethnic groups. Int J Dent. 2014; 2014:146028.

17. Vadavadagi SV, Kumari KH, Choudhury GK, et al. Prevalence of tooth shade and its correlation with skin colour-a cross-sectional study. J Clin Diagn Res. 2016;10:72-74.

18. Nourbakhsh M, Mousavinejad N, Adli AR, et al. Relationship between natural tooth shade and skin colour. Eur J Prosthodont Restor Dent. 2013;21:50-52.

19. Jahangiri L, Reinhardt SB, Mehra RV, Matheson PB. Relationship between tooth shade value and skin color: an observational study. J Prosthet Dent. 2002;87:149-152.

20. Lagouvardos PE, Tsamali I, Papadopoulou C, Polyzois G. Tooth, skin hair and eye colour interrelationships in greek young adults. Odontology. 2013;101:75-83.

21. Sabherwal RS, Gonzalez J, Naini FB. Assessing the influence of skin color and tooth shade value on perceived smile attractiveness. $J \mathrm{Am}$ Dent Assoc. 2009;140:696-705.

22. Labban $\mathrm{N}, \mathrm{Al}$-Otaibi $\mathrm{H}$, Alayed $\mathrm{A}$, et al. Assessing of the influence of gender and skin color on the preference of tooth shade in saudi population. Saudi Dent J. 2017;29:102-110.

23. Sitek A. Skin color parameters and Fitzpatrick phototypes in estimating the risk of skin cancer: a case-control study in the polish population. J Am Acad Dermatol. 2016;74:716-723.

24. Veeraganta SK, Savadi RC, Baroudi K, Nassani MZ. Differences in tooth shade value according to age, gender and skin color: a pilot study. J Indian Prosthodont Soc. 2015;15:138-141.

25. Lee KY, Setchell D, Stokes A, et al. Brightness (value) sequence for the Vita Lumin classic shade guide reassessed. Eur J Prosthodont Restor Dent. 2005;13:115-118.

26. Alshethri SE. Evaluation of color changes in the Vitapan classical shade guide after disinfection. Oper Dent. 2014;39:317-324.

27. Tashkandi E. Consistency in color parameters of a commonly used shade guide. Saudi Dent J. 2010;22:7-11.

28. Yamamoto $M$. The value conversion system and a new concept for expressing the shades of natural teeth. Quint Dent Technol. 1992;19: 2-9.

29. Niaz MO, Naseem M. Ethnicity and perception of dental shade esthetics. Int J Esthet Dent. 2015:10:286-298.

How to cite this article: Di Murro B, Gallusi G, Nardi R, Libonati A, Angotti V, Campanella V. The relationship of tooth shade and skin tone and its influence on the smile attractiveness. J Esthet Restor Dent. 2019;1-7. https://doi.org/ 10.1111/jerd.12543 